Accepted refereed manuscript of: Whittaker AC \& Gallagher S (2019) Caregiving alters immunity and stress hormones: a review of recent research. Current Opinion in Behavioral Sciences, 28, pp. 93-97. DOI:

https://doi.org/10.1016/j.cobeha.2019.02.002

(C) 2019, Elsevier. Licensed under the Creative Commons Attribution-NonCommercial-NoDerivatives 4.0 International http://creativecommons.org/licenses/by-nc-nd/4.0/

\title{
Caregiving alters immunity and stress hormones: a review of recent research
}

Anna C. Whittaker ${ }^{\mathrm{a}}$ (previously Phillips) \& Stephen Gallagher ${ }^{\mathrm{b}}$

aSchool of Sport, Exercise and Rehabilitation Sciences, University of Birmingham, Birmingham, United Kingdom.

bDepartment of Psychology, Health Research Institute, University of Limerick, Limerick, Ireland.

The authors have no conflicts of interest.

There is no funding source associated with this manuscript.

Word count: 2171

Address correspondence to: Anna C. Whittaker, School of Sport, Exercise and Rehabilitation Sciences, University of Birmingham, Birmingham B15 2TT, United Kingdom. Tel: +44 1214144398 E-mail: a.c.whittaker@bham.ac.uk 


\begin{abstract}
Older caregivers of dementia patients have been studied as a model of chronic stress influencing psychological and physical well-being and the function of the immune system. These immune decrements can relate to altered stress hormone levels, and caregivers show elevated or reduced cortisol compared to age-matched controls as well as dysregulation in other cortisol indices. Recently, this field has developed to examine a range of immune outcomes and cortisol measures in younger caregivers too, as well as other potential underlying mechanisms including genetic variation, acute stress reactivity, and inflammation. Several interventions have also been trialled to reduce the negative physical impact of caregiving stress. This paper reviews the latest developments in the field and gives suggestions for future research.
\end{abstract}

Keywords: caregiving; cortisol; immune function; stress; 


\section{Highlights}

- Caregiving effects are not restricted to older caregivers.

- Caregiving stress may accelerate immune ageing among younger caregivers.

- Positive effects of caregiving emerge where the burden is lower.

- Caregiver-patient dyads affect patient and caregiver immune/hormone outcomes.

- Interventions can reduce caregiver distress and immune/hormone dysregulation. 
Older caregivers of dementia patients have been studied as a model of chronic stress influencing psychological and physical well-being and the function of the immune system. Both innate and adaptive immunity are affected, for example, in older dementia caregivers natural killer (NK) cell function [1], antibody response to vaccination [2] and wound healing [3] is impaired in caregivers when compared to age and sex -matched controls. Caregiving is considered a model of chronic stress due to general extended nature of the caregiving period from months to years and the complexity of what is known as caregiver burden, describing the physical, emotional, and social impact of the stress of care-giving. This is well established as having a serious effect on psychological wellbeing and physical health among caregivers when compared to matched non-caregiving controls [4]. However, the caregivercare recipient relationship is dyadic, thus the caregiver can influence the health and wellbeing of the recipient as well as being influenced by the recipient's health and behaviour, for example, both members of such dyads can show increased depressive symptoms and sleep problems compared to controls [5]. Further, the caregiver-recipient relationship does not exist in a vacuum, thus It is important to examine the psychosocial factors and circumstances surrounding caregiving and protective roles which may interact and contribute to dysregulated immunity rather than treating caregiving as homogenous static stressor.

\section{Effects of caregiving among younger caregivers - specific aspects of caregiver distress}

Recent developments in caregiving research have focused on a range of immune markers, and younger caregiver populations such as parents. These show that younger caregivers are diverse and do not always display the immune decrements seen in older caregivers. For example, parental caregivers for children with developmental disabilities were no more likely than controls to be seropositive for the latent virus cytomegalovirus (CMV), nor show poorer virus control if seropositive [6]. One could conclude that in the absence of immunosenescence, not all aspects of immunity are compromised, but it may be that specific aspects of caregiving not just the role per se are important influencers of immunity. For example, particular aspects of the caregiving experience may influence or accelerate immune ageing as studies showed increased caregiver burden in young and older caregivers [7] and parental stress in younger caregivers [8] related to poorer neutrophil phagocytosis (ingestion) and superoxide production [7] and more advanced T cell differentiation [8], both associated with immune ageing. Further, these findings highlight potential mechanisms by which some caregivers might report poorer health given that poor sleep quality [7], and less exercise, smoking, and an unbalanced diet, as well as higher BMI [6] related to immune outcomes across studies.

\section{Caregiving and inflammation}


The impact of caregiving on other roles and other aspects of immunity has been demonstrated in studies of inflammatory markers (such as cytokine immune messengers and C-reactive Protein (CRP). For example, low leisure activities enjoyment/satisfaction among Alzheimer's caregivers were associated with higher inflammatory cytokine levels, but not with CRP or IL-6 or with depression [9]. This suggests that the absence of positive affect not just the presence of negative affect influences inflammation, thus may be an appropriate target for behavioural interventions. This is important given that inflammation plays a key role in various diseases [10], and increases with ageing [11], meaning that the impact of caregiving stress may be even greater in older caregivers. However, such consequences are not limited to older caregivers, but may be more apparent in those reporting high burden, or during specific stressful periods. For example, increased inflammatory cytokines have been shown to be specific to males with anxiety, high BMI, disrupted schedules, ages 30-39 years with high burden [12] and those with high grief in the presence of blame and anger [13] or in the 6-months post-diagnosis of a child with cancer in caregiving mothers [14], a particularly stressful period. In support of this idea about stressful time periods, not all markers of inflammation were raised among long-term caregivers [15] and in caregivers with low caregiving burden allostatic load (incorporating inflammatory markers) was lower than non-caregivers [16]; potentially indicating lower distress at less stressful periods. These studies also indicate a benefit for caregivers with multiple social roles or lower burden; supportive evidence showed that having multiple roles in later life, including caregiving, related to lower levels of CRP [17]. However, the most robust association was with volunteer work, reiterating that caregiving can be beneficial if low burden, i.e. allowing time for other activities like volunteering. This underlines the importance of examining the psychosocial factors and circumstances surrounding caregiving.

\section{Caregiving and genetic variations}

One recently identified pathway to poorer immunity in caregivers is genetics, specifically single nucleotide polymorphisms (SNPs) of cytokine promotor genes. Variations in alleles for certain cytokine promotor genes (and other genes associated with inflammation) have been shown to relate to a range of important individual differences in caregivers of oncology patients such as poorer attentional function, a subtle indicator of cognitive change and important predictor of quality of life [18]; and morning or evening fatigue [19]; higher trait and state anxiety [20] and poorer quality of life [21]. Although other factors such as age, and comorbidities contributed to these psychosocial and behavioural outcomes, these studies corroborate reports of caregiving stress effects on systemic inflammation and reveal another mechanism by which caregiving can contribute to immune dysfunction. Another mechanism is gene transcription and expression; caregivers' monocytes showed increased expression of pro-inflammatory genes such as those bearing response elements for $\mathrm{NF} \kappa \mathrm{B}$, a pro-inflammatory transcription factor, as well as increased 
IL-6 production in vitro [15]. Some studies included patients not exclusively caregivers, but do indicate why certain individuals may be more at risk of adverse effects, and identify those most in need of additional intervention.

\section{The caregiver-care recipient dyad}

The impact of psychosocial and behavioural factors within caregivers can be considered a proxy for patient distress, having effects on patients' immune outcomes. In cancer patients undergoing stem cell transplantation to replace blood cell progenitors that can then differentiate into new healthy cells, high caregiver depression, avoidance, and poor sleep have been observed [22]. Where caregivers had better objective and subjective sleep quality, patients experienced earlier neutrophil engraftment, a marker of faster stem cell transplant success [23]. Such studies emphasise the importance of wellbeing within this dyadic relationship, particularly during key periods of vulnerability where the impact of distress is higher for both caregivers and care recipients, but equally, interventions may be most effective.

\section{Cortisol and immunity}

The stress response system, specifically the stress hormone cortisol, which generally results in down-regulation of immunity and inflammation [11] may be a mechanism underlying caregiving effects on immunity [24]. During stress, the hypothalamic-pituitary-adrenal (HPA) axis is triggered and provokes the systemic release of glucocorticoids (GCs), which have anti-inflammatory and immunosuppressive actions on immune cells via their receptor. For example, cortisol influences the function of effector cells like monocytes and macrophages while increasing their phagocytic potential; moreover, cortisol abrogates the production of pro-inflammatory cytokines.

\section{Caregiving and cortisol}

Several studies have examined basal cortisol (i.e. at rest not in response to an acute stressor) as an index of chronic stress. In caregivers of cancer patients undergoing hematopoietic stem cell transplant treatment serum cortisol concentrations were lower at compared to controls [25], suggesting dysregulation. However, social support and depression accounted for group differences in hair cortisol between caregivers of dementia patients and controls [26] implying, as argued above, it is not always caregiving per se that is damaging but specific aspects of the caregiving experience. Further, these studies indicate that caregivers have lower basal cortisol, often associated with inflammatory disease states [27]. 
The diurnal patterning of hormone secretion across the day, such as in daily diary studies, provides important clues to HPA axis dysregulation and is associated with health outcomes. These studies also mainly support low cortisol among caregivers, and specifically among those experiencing specific negative aspects of caregiving such as individuals used less respite and than caregivers who used more respite [28]; or who expressed higher feelings of anger [29]. Similarly, a smaller or flatter cortisol awakening response (CAR), the brisk increase of cortisol levels within 20-30 min after awakening, was associated with higher depression in caregivers [29] and with caregiving per se among younger caregivers of children with Autism [30], although not in all studies [31]. However, it is possible that other markers of HPA axis regulation are more sensitive to psychosocial stressors. For example, using intra-individual cortisol variability (ICV) as an index of HPA axis regulation within caregivers of hematopoietic stem cell transplant patients, a greater ICV was associated with poor mental health, with no associations observed for other cortisol HPA axis indices (area under the curve, diurnal decline or CAR) [31]. Together, these studies confirm dysregulation of the HPA axis in both older and young caregivers and suggest that factors such as social support and depression are important for regulatory processes.

Another important advancement is studies demonstrating altered glucocorticoid sensitivity, which influences inflammatory processes. In longitudinal studies, caregivers showed lower glucocorticoid receptor (GR) signaling [15] or increased glucocorticoid resistance [14] where no changes in cortisol itself were observed compared to controls. This implies that is it not the bioavailability of cortisol nor its receptor that is affected by caregiving but rather GR signalling/responsivity. Changes in distress over time among mothers of children newly diagnosed with cancer correlated with changes in glucocorticoid resistance over a 12-month period, particularly in those with increasing depression levels [14], which again illustrates the impact of the emotional response to caregiving not just the caregiving role itself.

A commonly used model of the consequences of stress for cortisol is acute stress reactivity, generally studied in the context of a laboratory stress task as a predictor of future disease risk in those with exaggerated or low/blunted responses [32]. For example, parental caregivers for individuals with eating disorders, showed blunted salivary antibody or immunoglobulin A ( $\operatorname{sg} \mathrm{A})$, and cortisol to acute stress, indicating dysregulated stress-induced responses, as well as lower slgA levels overall [33]. This builds on previous literature where a blunted cardiovascular response to acute stress was associated with an impaired antibody response to vaccination [34]. Moderate cortisol reactivity is likely the most adaptive response, and in a social support manipulation, caregivers who were given high personcentred support, rather than medium-person centred support, from a member of their social network while they discussed the challenges of caregiving were found to display lower to moderate cortisol reactivity [35]. This emphasises social support as a further moderating factor of caregiver health. 


\section{Interventions to ameliorate negative effects of caregiving}

Early interventions have shown some promising effects for immune and stress hormone outcomes but are sometimes hampered by bias such as non-randomisation. The group studying caregiver-patient dyads in the context of stem cell transplantation tested a stress management intervention for caregivers [22]. Despite no overall reduction in inflammatory markers, caregivers who received the intervention showed down-regulation of expression of transcription pathways associated with inflammation, and reduced depression and anxiety levels [22]. Although this sub-analysis suffered from a small sample, precluding adjustment for confounders, it elucidates the role of genes at the transcription level, and helps explain the inflammatory effects observed in long-term caregivers reporting high distress or burden.

For cortisol, some studies $[28,29]$ adopted naturalistic interventions such as respite. Among those with intentional interventions, there are mixed results; there was no difference in basal cortisol levels [36] or the CAR [22] compared to controls, however, a service dog intervention for families of children with Autism did show some evidence of improved HPAaxis regulation [37]. Where caregivers were required to do activities with their carerecipient [36], it may have left little time for personal respite and support for the caregiver perhaps explaining the null effects in certain studies. Given that some HPA-axis indices (e.g., ICV) have been shown to be more sensitive to psychosocial factors this may be a fruitful line of enquiry for future intervention research.

\section{Conclusion}

The key messages that have emerged from this review of the recent caregiving literature are that negative effects on immunity and cortisol are not restricted to older spousal caregivers, however, caregiving stress may accelerate immune system ageing among younger caregivers and dysregulate their HPA axis. Further, caregiver-patient dyads are important and can affect patient as well as caregiver outcomes. Also, caregiving effects are not homogenous but vary with the caregiving experience such as greater effects in the presence of high distress or burden. However, where burden is lower and caregivers have a range of roles, positive effects of caregiving can emerge, this contrast is shown in Figure 1. More recent genetic research has revealed that carriers of particular polymorphisms of certain cytokine genes may be more at risk of poor emotional outcomes. However, stress management can reduce caregiver distress and down-regulate transcription of proinflammatory genes, and interventions such as a service dog for families can alter HPA axis regulation. Thus, recent evidence suggests a number of important psychosocial factors to attend to when intervening with caregivers as well as some potential means to reduce the negative impact of caregiving on stress hormones and immunity.

[Insert Figure 1 about here] 
Figure 1: Effects of caregiving on HPA-axis and immune outcomes along with potential mechanisms involved in vulnerability and resiliency 


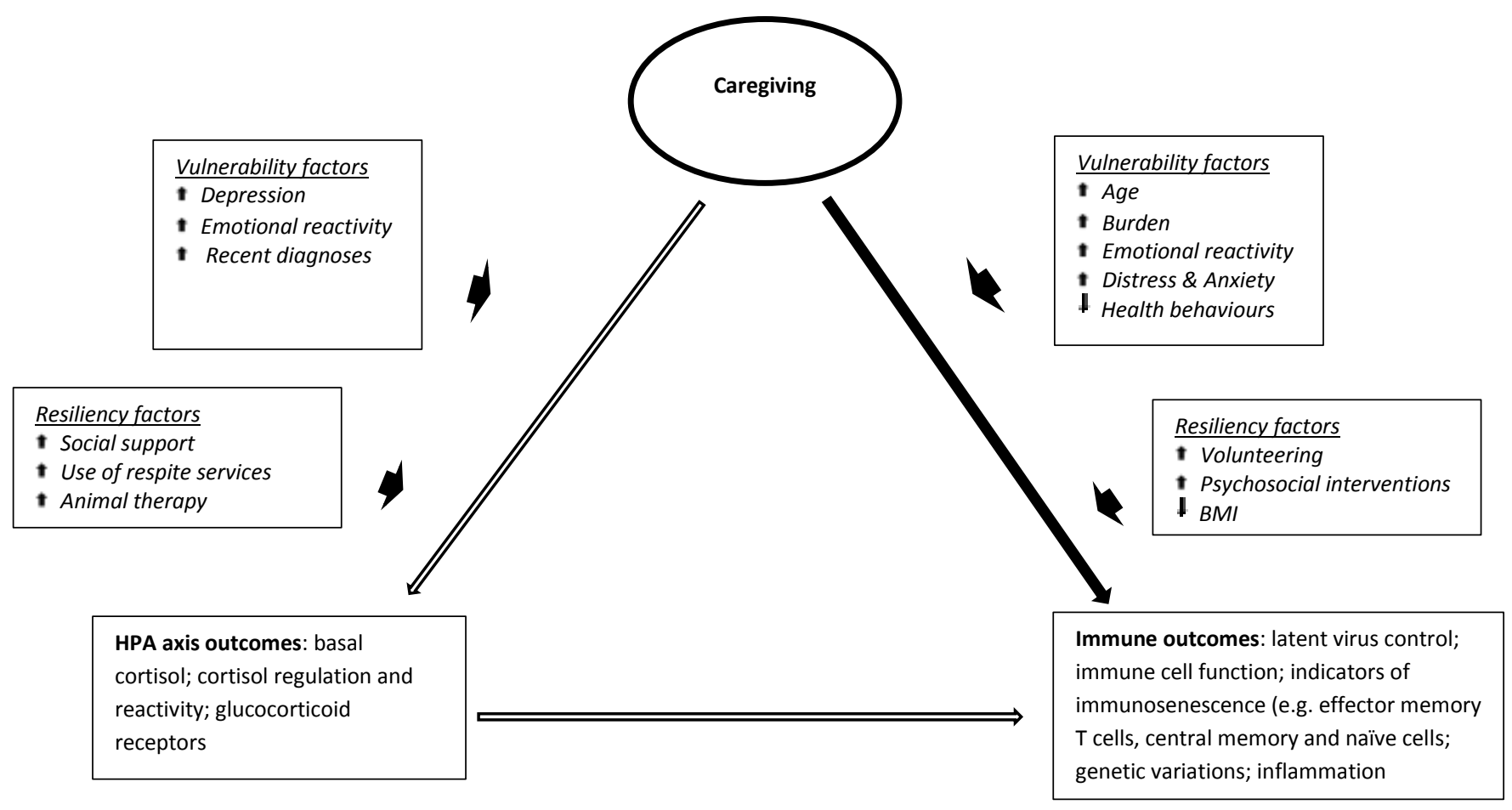




\section{References}

1. Esterling BA, Kiecolt-Glaser JK, Glaser R: Psychosocial modulation of cytokineinduced natural killer cell activity in older adults. Psychosom Med 1996, 58:264-272.

2. Glaser R, Sheridan JF, Malarkey W, MacCallum RC, Kiecolt-Glaser JK: Chronic stress modulates the immune response to a pneumococcal pneumonia vaccine.

Psychosom Med 2000, 62:804-807.

3. Kiecolt-Glaser JK, Marucha PT, Malarkey WB, Mercado AM, Glaser R: Slowing of wound healing by psychological stress. Lancet 1995, 346:1194-1196.

4. Pinquart $M$, Sorensen S: Differences between caregivers and noncaregivers in psychological health and physical health: a meta-analysis. Psychol Aging 2003, 18:250-267.

5. Bishop MM, Beaumont JL, Hahn EA, Cella D, Andrykowski MA, Brady MJ, Horowitz MM, Sobocinski KA, Rizzo JD, Wingard JR: Late effects of cancer and hematopoietic stem-cell transplantation on spouses or partners compared with survivors and survivor-matched controls. J Clin Oncol 2007, 25:1403-11.

6. Vitlic A, Phillips AC, Gallagher S, Oliver C, Lord JM, Moss P: Anticytomegalovirus antibody titres are not associated with caregiving burden in younger caregivers. $\mathrm{Br} J$ Health Psychol 2015, 20:68-84.

7. Vitlic A, Lord JM, Taylor AE, Arlt W, Bartlett DB, Rossi A, Arora-Duggal N, Welham A, Heald $M$, Oliver $C$, et al.: Neutrophil function in young and old caregivers. Br J Heal Psychol 2016, 21:173-189.

8. Prather AA, Epel ES, Portela Parra E, Coccia M, Puterman E, Aiello AE, Dhabhar FS: Associations between chronic caregiving stress and $\mathrm{T}$ cell markers implicated in immunosenescence. Brain Behav Immun 2018, doi:10.1016/j.bbi.2018.06.019.

9. von Känel R, Mausbach BT, Mills PJ, Dimsdale JE, Patterson TL, Ancoli-Israel S, Ziegler MG, Allison M, Chattillion EA, Grant I: Longitudinal Relationship of Low Leisure Satisfaction but not Depressive Symptoms With Systemic Low-Grade Inflammation in Dementia Caregivers. Journals Gerontol Ser B Psychol Sci Soc Sci 2014, 69:397-407.

10. Vitaliano PP, Zhang J, Scanlan JM: Is Caregiving Hazardous to One's Physical Health? A Meta-Analysis. Psychol Bull 2003, 129:946-972.

11. Phillips AC, Burns VE, Lord JM: Stress and exercise: Getting the balance right for aging immunity. Exerc Sport Sci Rev 2007, 35:35-39.

12. Sherwood PR, Price TJ, Weimer J, Ren D, Donovan HS, Given CW, Given BA, Schulz R, Prince J, Bender $\mathrm{C}$, et al.: Neuro-oncology family caregivers are at risk for systemic inflammation. J Neurooncol 2016, 128:109-118.

13. Saban KL, Mathews HL, Collins EG, Hogan NS, Tell D, Bryant FB, Pape TL, Griffin JM, Janusek LW: The Man I Once Knew: Grief and Inflammation in Female Partners of Veterans With Traumatic Brain Injury. Biol Res Nurs 2016, 18:50-59.

14. Walsh CP, Ewing LJ, Cleary JL, Vaisleib AD, Farrell CH, Wright AGC, Gray K, Marsland $\mathrm{AL}$ : Development of glucocorticoid resistance over one year among mothers of children newly diagnosed with cancer. Brain Behav Immun 2018, 69:364-373.

15. Miller GE, Murphy ML, Cashman R, Ma R, Ma J, Arevalo JM, Kobor MS, Cole SW: Greater inflammatory activity and blunted glucocorticoid signaling in monocytes of chronically stressed caregivers. Brain Behav Immun 2014, 41:191-199.

16. Dich N, Lange T, Head J, Rod NH: Work stress, caregiving, and allostatic load: 
prospective results from the Whitehall II cohort study. Psychosom Med 2015, 77:539-547.

17. Kim S, Ferraro KF: Do productive activities reduce inflammation in later life? Multiple roles, frequency of activities, and C-reactive protein. Gerontologist 2014, 54:830-839.

18. Merriman JD, Aouizerat BE, Langford DJ, Cooper BA, Baggott CR, Cataldo JK, Dhruva A, Dunn L, West C, Paul SM, et al.: Preliminary evidence of an association between an interleukin 6 promoter polymorphism and self-reported attentional function in oncology patients and their family caregivers. Biol Res Nurs 2014, 16:152-159.

19. Dhruva A, Aouizerat BE, Cooper B, Paul SM, Dodd M, West C, Wara W, Lee K, Dunn $L B$, Langford DJ, et al.: Cytokine gene associations with self-report ratings of morning and evening fatigue in oncology patients and their family caregivers. Biol Res Nurs 2015, 17:175-184.

20. Miaskowski C, Cataldo JK, Baggott CR, West C, Dunn LB, Dhruva A, Merriman JD, Langford DJ, Kober KM, Paul SM, et al.: Cytokine gene variations associated with trait and state anxiety in oncology patients and their family caregivers. Support Care Cancer 2015, 23:953-965.

21. Alexander K, Cooper B, Paul SM, West C, Yates P, Kober KM, Aouizerat BE, Miaskowski $C$ : Evidence of associations between cytokine gene polymorphisms and quality of life in patients with cancer and their family caregivers. Oncol Nurs Forum 2014, 41:E267-81.

22. Laudenslager ML, Simoneau TL, Kilbourn K, Natvig C, Philips S, Spradley J, Benitez P, Mcsweeney P, Mikulich-Gilbertson SK: A randomized control trial of a psychosocial intervention for caregivers of allogeneic hematopoietic stem cell transplant patients: Effects on distress. Bone Marrow Transplant 2015, 50:1110-1118.

23. Sannes TS, Mikulich-Gilbertson SK, Natvig CL, Brewer BW, Simoneau TL, Laudenslager ML: Caregiver Sleep and Patient Neutrophil Engraftment in Allogeneic Hematopoietic Stem Cell Transplant. Cancer Nurs 2018, 41:77-85.

24. Bauer ME, Vedhara K, Perks P, Wilcock GK, Lightman SL, Shanks N: Chronic stress in caregivers of dementia patients is associated with reduced lymphocyte sensitivity to glucocorticoids. J Neuroimmunol 2000, 103:84-92.

25. Bevans MF, Ross A, Wehrlen L, Klagholz SD, Yang L, Childs R, Flynn SL, Remaley AT, Krumlauf $M$, Reger RN, et al.: Documenting stress in caregivers of transplantation patients: initial evidence of HPA dysregulation. Stress 2016, 19:175-184.

26. Stalder T, Tietze A, Steudte S, Alexander N, Dettenborn L, Kirschbaum C: Elevated hair cortisol levels in chronically stressed dementia caregivers.

Psychoneuroendocrinology 2014, 47:26-30.

27. Edwards LD, Heyman AH, Swidan S: Hypocortisolism: An Evidence-based Review. Integr Med 2011, 10:26-33.

28. Liu Y, Almeida DM, Rovine MJ, Zarit SH: Care Transitions and Adult Day Services Moderate the Longitudinal Links between Stress Biomarkers and Family Caregivers' Functional Health. Gerontology 2017, 63:538-549.

29. Leggett AN, Zarit SH, Kim K, Almeida DM, Klein LC: Depressive Mood, Anger, and Daily Cortisol of Caregivers on High- and Low-Stress Days. J Gerontol B Psychol Sci Soc Sci 2015, 70:820-829.

30. Foody C, James JE, Leader G: Parenting Stress, Salivary Biomarkers, and Ambulatory Blood Pressure: A Comparison Between Mothers and Fathers of Children with 
Autism Spectrum Disorders. J Autism Dev Disord 2015, 45:1084-1095.

31. Sannes TS, Mikulich-Gilbertson SK, Natvig CL, Laudenslager ML: Intraindividual Cortisol Variability and Psychological Functioning in Caregivers of Hematopoietic Stem Cell Transplant Patients. Psychosom Med 2016, 78:242-247.

32. Carroll D, Ginty AT, Whittaker AC, Lovallo WR, de Rooij SR: The behavioural, cognitive, and neural corollaries of blunted cardiovascular and cortisol reactions to acute psychological stress. Neurosci Biobehav Rev 2017, 77:74-86.

33. Romero-Martínez Á, Moya-Albiol L: Stress-Induced Endocrine and Immune Dysfunctions in Caregivers of People with Eating Disorders. Int J Environ Res Public Health 2017, 14:1560.

34. Phillips AC, Carroll D, Burns VE, Drayson M: Cardiovascular activity and the antibody response to vaccination. J Psychosom Res 2009, 67:37-43.

35. Faw MH: Supporting the supporter: Social support and physiological stress among caregivers of children with severe disabilities. J Soc Pers Relat 2018, 35:202-223.

36. Hirano A, Umegaki $H$, Suzuki $Y$, Hayashi T, Kuzuya M: Effects of leisure activities at home on perceived care burden and the endocrine system of caregivers of dementia patients: a randomized controlled study. Int Psychogeriatr 2016, 28:261268.

37. Fecteau SM, Boivin L, Trudel M, Corbett BA, Harrell Jr. FE, Viau R, Champagne N, Picard F: Parenting stress and salivary cortisol in parents of children with autism spectrum disorder: Longitudinal variations in the context of a service dog's presence in the family. Biol Psychol 2017, 123:187-195.

- Sherwood et al (2016) study focuses on younger caregivers of cancer patients and shows an important link with caregiving stress and inflammation as well as showing that caregiving effects are not homogeneous but that effects may relate to specific behavioural and emotional responses such as anxiety, or life disruption.

- Walsh et al (2018) are the first to demonstrate that chronic stress influences the development of glucocorticoid resistance by downregulating the sensitivity of glucocorticoid receptors, which corresponded with changes in psychological distress.

- Romero-Martinez et al (2017) study is the first to examine this specific group of caregivers. It showed pervasive effects on the stress responses of the immune system and HPA axis as well as down-regulation of oral immunity.

- Faw (2018) study shows the importance of social factors for caregiver cortisol responses during stress. However, not all social interactions are equal with greater reductions in cortisol reactivity correlating with high person-centred but not medium-centred supportive interactions.

- Fecteau et al (2017) study found that a service dog provided to families caring for children with autism not only reduced their psychological distress but it also improved regulation of their hypothalamic-pituitary-adrenal (HPA) axis secretion of cortisol. 